\title{
Bedarfsanalyse der Fachsprachenlehrerausbildung und Problemlösungsmöglichkeiten im berufsorientierten Sprachunterricht
}

\author{
Neli Akhvlediani \\ Associate Professor \\ Batumi Shota Rustaveli State University \\ Georgia
}

\begin{abstract}
In den beiden letzten Jahrzehnten ist in Georgien der Bedarf an Fremdsprachenkenntnissen im Beruf schnell gestiegen. Dementsprechend wurden das Interesse an der fachsprachlichen Thematik sowie die Bedeutung des Fachsprachenunterrichts zugenommen.Normalerweise müssen die Lehrende im Fach ihrer jeweiligen Zielgruppe Kenntnisse aufweisen. Diese sind aber nicht immer vorhanden. die Hochschullehrer/Innen, die Fachsprachen an der ShotaRustaveli Universität Batumi unterrichten, haben meistens keine Ausbildung als FACHsprachenlehrer/Innen erhalten. Im Jahr 2013 wurde an der ShotaRustaveli Universität Batumi das Zentrum für Sprachen und Informationstechnologie gegründet. Leider spielen Forschungsaktivitäten im Sprachenzentrum bisher noch eine untergeordnete Rolle. Zielgerichtete empirische Untersuchungen zu hochschulspezifischen fremdsprachlichen Lern- und Lehrprozessen können Grundlage für eine Erhöhung der Qualität von Sprachlernangeboten an Hochschulen sein.Im vorliegenden Artikel werden die obengenannte Problematik sowie Problemlösungsmöglichkeiten im berufsbezogenen Sprachunterricht beschrieben.
\end{abstract}

Schlüsselwürter: hochschulspezifische Fachsprachen; fachorientierte Lehrerfortbildung; berufsorientierter Sprachunterricht.

\section{Einleitung}

Die Motivation für den vorliegenden Artikel leitet sich an dem Forschungsdefizit im Bereich Fachsprachenunterricht ab. Im Jahr 2013 wurde an der ShotaRustaveli Universität Batumi das Zentrum für Sprachen und Informationstechnologie gegründet. Im Laufe meiner Lehrtätigkeit und Leitung des Sprachenzentrums der Staatlichen ShotaRustaveli Universität Batumi ist mir die Problematik des Fachsprachenunterrichts bewusst geworden. Für die Fremdsprachendidaktiker in Georgien ist die Schule der einzige Lernort, der von ihnen untersucht wurde. Hochschulen und Universitäten sind bisher nicht untersucht worden. Das bedeutet, dass die institutionell geförderte Forschung zu den wünschenswerten und notwendigen Veränderungen geführt werden muss.

\section{Forschungsziel}

Das Ziel der Forschung ist, die Lehrpläne bei höherer Bildung zu erneuern und Curricula für den fachbezogenen Fremdsprachenunterricht zu erarbeiten;

Um das oben genannte Ziel zu erreichen ist es wichtig, die Antwort auf folgende Fragen zu finden:

1. Was für ein institutionelles System existiert an der Universität Batumi, innerhalb deren fachorientierter Fremdsprachenunterricht stattfinden soll?

2. Welche Anforderungen soll man an die Lehrenden stellen, um über eine ausreichende (fach)fremdsprachliche Kompetenz für das Sprachenzentrum zu verfügen?

\section{Gemeinsprache-Fachsprache}

Bevor ich darauf eingehen würde, wie die Fachsprache definiert werden kann, will ich den Begriff Gemeinsprache klären. Für beide Begriffe gibt es viele Definitionen. Was den Begriff Gemeinsprache anbelangt, möchte ich hier die Definition von Dieter Möhn und Roland Pelka (Möhn/Pelka, 1984:141) zitieren, nach der die Gemeinsprache die Variante der Gesamtsprache ist, über die mehr oder weniger alle Sprachteilhaber in gleicher Weise verfügen und deren schriftlicher oder mündlicher Gebrauch in öffentlichen wie privaten Situationen primär der Kommunikation über allgemeine Inhalte des täglichen, gesellschaftlichen und privaten Lebens dient. 
Was den Begriff Fachsprache betrifft, möchte ich die Definition von Lothar Hoffmann anführen. „Die Fachsprache ist die Gesamtheit der sprachlichen Mittel, die in einem fachlich begrenzbaren Kommunikationsbereich verwendet werden. Diese sprachlichen Mittel dienen der Verständigung zwischen den Menschen, die in diesem Bereich tätig sind und der Popularisierung der fachlichen Inhalte sowie dem Kontakt zu bestimmten Nicht-Fachleuten.“ (Hoffmann, 1976:170)

\section{Allgemeinsprachlicher Unterricht -Fachsprachenunterricht}

Im Mittelpunkt des Artikels steht der (fach) fremdsprachlicher Unterricht. Welche Funktion trägt der fachsprachlicher Unterricht und wie unterscheidet man ihn vom allgemeinsprachlichen Unterricht?,Der allgemeinsprachliche Unterricht ist primär auf Kommunikationsfähigkeit in der Alltagsituation ausgerichtet. Das bedeutet, daß er versucht, sprachliche Handlungsfähigkeit in vornehmlich dialogischen Situationen zu vermitteln. Dabei geht es um Strategien sprachlicher Behauptung in Argumentationssituationen im Alltag unter Anwendung der zahlreichen dort gebräuchlichen Kommunikationsverfahren“. (Buhlmann/ Fearns 2000: 81)

„Der allgemeinsprachliche Unterricht ist nicht auf Inhalte ausgerichtet, an denen sich Arbeitsstrategien (study) entwickeln lassen. Der Fachunterricht setzt diese voraus. Aus dieser Gegenüberstellung wird deutlich, daß es von allgemeinsprachlichen Unterricht nicht ohne weiteres einen direkten Weg zum Überleben im Fachunterricht gibt: Es werden nicht nur Fertigkeiten entwickelt, die im Unterricht nicht benötigt werden, sondern auch solche, die unvereinbar mit den tatsächlich benötigten sind bzw. ihre Entwicklung verhindern können. Der allgemeinsprachliche Unterricht entwickelt jedenfalls nicht automatisch die Fertigkeiten, die im Fachunterricht erforderlich sind und die dort vorausgesetzt werden“. (Buhlmann/ Fearns 2000: 82)

„Der Fachsprachenunterricht bildet also gewissermaßen eine Brücke zwischen allgemeinsprachlichem Unterricht und dem Fachuterricht. Es kann die Lerner auf den Fachunterricht vorbereiten, einmal indem er kompensatorische Strategien im Bereich der Informationsentnahme und Textproduktion aufbaut, zum anderen indem er Denkelemente zur Verfügung stellt und damint den Auf- bzw. Ausbau von Denkstrukturen ermöglicht, zum dritten, indem er die Lerner mit bestimmten stilistischen Eigentümlichkeiten der Kommunikation im Fach bekannt macht (Präzision, Differenziertheit, Hierarchisierung, Ökonomie etc.)“ (Buhlmann/ Fearns 2000: 85).

\section{Sprachlehrforschung}

Da ich mich sehr für die Ausbildungsforschung der FremdsprachenlehrerInnen an den Hochschulen interessiere, gehe ich auf die Sprachlehrforschung und Fachsprachendidaktik ein. "Sprachlehrforschung ist als Abkürzung von "Sprachlehr- und Sprachlernforschung" zu verstehen, denn trotz des eigentlich irreführenden Namens geht es der Sprachlehrforschung um beides: das Lehren und das Lernen von Fremdsprachen. Beide Prozesse sind eng miteinander verknüpft, obwohl natürlich gilt, daß das, was im Fremdsprachenunterricht gelehrt wird, keinesfalls identisch ist mit dem, was darin gelernt wird. Wenn dies stimmt, dann setzt die "Sprachlehrforschung" eine "Sprachlernforschung" voraus und zwar in dem Sinne, daß ein begründetes Konzept des Fremdsprachenlehrens eine begründete Theorie des Fremdsprachenlernens voraussetzt" (Edmonson/Heuse, 2011:3). Eine der wichtigsten Aufgaben der Sprachlehrforschung ist die Ausbildungsforschung der FremdsprachenlehrerInnen an den Hochschulen (Hans-Jurgen Krumm 1997: 109). Hans-Jurgen Krumm plädiert dafür, die Hochschulen als Forschungsfeld für die Sprachlehrforschung zurückzugewinnen. Der Autor des Artikels: "Ausbildung und Ausbildungsforschung als Aufgaben von Sprachlehrforschung und Fremdsprachendidaktik" wünscht die systematische Curriculumentwicklung und -evaluation sowie Begleituntersuchungen $\mathrm{zu}$ universitären Sprachlehrveranstaltungen (Hans-Jurgen Krumm 1997: 112).Klaus- Dieter-Baumann beschreibt in seinem Artikel: "Die Entwicklung eines integrativen Fachsprachenunterrichts- eine aktuelle Herausforderung der angewandten Linguistik" die Bedeutung von Fachsprachenforschung und Fachsprachendidaktik für die Konzeption eines integrativen Fachsprachenunterrichts. In der Zusammenfassung stellt er fest, dass die Befähigung zur effizienten Bewältigung von kommunikativen Handlungssituationen im Fach durch einen integrativen fachsprachlichen (Fremd-)Sprachenunterricht erfolgen kann (vgl. Klaus-Dieter Baumann, 2000:170). Fachsprachliche Ausbildung in vielen Ländern an Fremdspracheninstituten, Universitäten und Schulen nicht fremd sind. , Als Konsequenz der allgemein beruflichen wie der wissenschafltichen Entwicklung sehen wir uns jerdenfalls der Tatsache gegenüber, dass solide und anwendungsbereite Kenntnisse in international führenden Sprachen für Hochschulabsolventen zu den notwendigen Elementen ihrer Berufsbefähigung gehören. (Meyer, 2000: 32). 


\section{Forschungsmethode}

Über welche spezifischen sprachlichen Kenntnisse und Fähigkeiten sollten FremdsprachenlehrerInnen verfügen, um ihren Beruf effektiv ausüben zu können. Darüber hinaus ist zu hinterfragen, in wieweit die hochschulische sprachpraktische Ausbildung über die Ziele des allgemeinen Sprachunterrichts hinausgeht, und die spezifischen sprachlichen Bedürfnisse angehender FremdsprachenlehrerInnen berücksichtigt.Im Hinblick auf die Zielsetzung, möglichst genaue und brauchbare Informationen bereitzustellen, sowie dessen Gesamtproblematik zum ersten Mal zu erfassen, wurde die Entscheidung getroffen, in relativ kurzer Zeit mehrere Personen zu befragen. Darüber hinaus sind durch eine Befragung exakt quantifizierbare Ergebnisse möglich, auf deren Grundlage statistische Zusammenhänge aufgezeigt werden können.

Ziel der vorliegendenquantitativenUntersuchung ist dieErforschung der gegenwärtigen Situation des (fachsprachlichen) Unterrichts und der Unterrichtssituation an der ShotaRustaveliUniveristät Batumi sowie der didaktisch-methodischen Vorstellungen und Orientierungen bei den Hochschullehrern. Zu diesem Zweck wurde an der Universität Batumi eine empirischeUntersuchung über HochschullehrerInnen im Sprachenzentrum durchgeführt. Die Forschungsdaten wurden schriftlich mittels Fragebögen erhoben. Es wurden folgende Themen behandelt:

- Fachsprachenlehrerausbildung

- Möglichkeiten der fachorientierten Lehrerfortbildung

- Unterrichtskonzept für Fachsprachenunterricht

- fachorientierte Lern- und Lehrmaterialien

- fachliche Wissensgrunde der an der Universität Batumi beschäftigten Lehrkräfte

- Curriculum für den fachbezogenen Fremdsprachenunterricht

\section{Ergebnisse der quantitativen Untersuchung}

An Hand der an der ShotaRustaveli Universität Batumi geführten quantitativen Forschung möchte ich einen Überblick über die Probleme im spezifischen Lern- und Lehrkontext an der Universität Batumi schaffen. Im Rahmen der Untersuchung wurden 77 HochschullehrerInnen (Im Sprachenzentrum der ShotaRustaveli Universität Batumi dominieren die weiblichen Lehrkräfte, -76 Frauen und 1 Mann) schriftlich befragt. An der Befragung haben 7 Germanistinnen, 4 Romanistinnen, 5 Russistinnen und 61 Anglisten teilgenommen. Das Alter der Befragten schwankte von 25 bis 60 Jahren. Die Befragung wurde in der georgischen Sprache durchgeführt, weil die Probandengruppe fremdsprachlich heterogen war.

Die gestellten Fragen wurden wie folgt beantwortet:

- Verfügen Sie über eine Zusatzqualifikation für den Fachsprachenunterricht?

- von 77 Probanden nur zwei verfügen über Zusatzqualifikation (1- Medizin, 1- Betriebswirtschaft).

- Haben Sie an den fachsprachenorientierten Lehrerfortbildungen teilgenommen?

- $18 \%$ der Befragten haben berufsbezogene Fortbildungen gemacht. 82\% der Befragten haben keine fachsprachliche Weiterbildung gemacht.

- Haben Sie ein Unterrichtskonzept für den Fachsprachenunterricht?

- $16 \%$ der Befragten haben ein berufsbezogenes Unterrichtskonzept erarbeitet und $84 \%$ der Probanden verfügen über kein Konzept.

- Werden für den Unterricht die Lehrwerke verwendet, die direkt für die Zielgruppe entwickelt wurden? - 28\% der Befragten verwenden die für die Zielgruppe entwickelten Lehrwerke und $72 \%$ der Probanden benutzen allgemeinsprachliche Lehrwerke.

- Über welches Niveau verfügen die Studierenden am Anfang des Studiums?

- 14\% der Studierenden haben das Sprachniveau A1. Das Sprachniveau A2 dominiert mit 65\%, B1 haben 14\% der Student/Innen und nur 7\% der Student/Innen haben B2.

- Wie alt sind die Studierenden am Anfang des Studiums?

- 78\% der Student/Innen sind zwischen 18 und 20 Jahre alt und 22\% der Student/Innen sind im Alter von 21 bis 27 Jahren.

- Unterrichten Sie in ALLEN Fakultäten eine fachbezogene Fremdsprache?

- 100\% der Probanden unterrichten nicht in allen Fakultäten. Sie haben kommentiert, dass fachbezogene Fremdsprache an der Wirtschafts-, Sozial- und juristischen Fakultäten unterrichtet wird; ebenso an der Fakultät für Tourismus. Nach Angaben des Studienjahres 2014-2015 gibt es insgesamt 14 Gruppen: 
- 4 Gruppen an der wirtschafts- und sozialwissenschaftliche Fakultät;

- 4 Gruppen an der juristischen Fakultät;

- 6 Gruppen an der Fakultät für Tourismus.

- Kann der Hochschullehrer ohne Fachwissen problemlos eine Fachsprache unterrichten?

- 11\% der Befragten finden, dass sie ohne Fachkenntnisse eine Fachsprache unterrichten können und 89\% der Probanden sind anderer Meinung.

- Haben Sie Probleme bei der Erklärung der neuen Fachtermini?

- 27\% der Befragten haben Schwierigkeiten mit den Fachbegriffen; 11\% der Beteiligten können problemlos mit den neuen Fachbegriffen umzugehen und 62\% der Befragten haben manchmal Schwierigkeiten mit berufsbezogenen Fachtermini.

\section{Zusammenfassung}

Anhand der empirisch fundierten Analyse wurde festgestellt, dass eine Vermittlung von fachorientierten Fremdsprachen im Hochschulbereich unter den gegenwärtigen Bedingungen nur erschwert möglich ist. Es ist offenbar, dass das Problem nicht in einer schlechten Ausbildung der Hochschullehrer/Innen liegt, sondern im Mangel an methodisch-didaktischen Konzeptionen des fachbezogenen Fremdsprachenunterrichts und der fehlenden Ausbildungsmöglichkeit als Fachsprachenlehrer. Ein weiterer Punkt wäre die Entwicklung der Curricula, die auf die jeweiligen Studiengänge zugeschnitten werden soll. In Bezug auf Fachsprachenunterricht konnte davon ausgegangen werden, dass die allgemeine Unterrichtssituation in Batumi den Anforderungen der modernen gesellschaftlichen und wirtschaftlichen Entwicklungen zumindest nicht gerecht wird. Aufgrund der Eindrücke, die ich während meiner Berufstätigkeit gesammelt habe, sowie des Hintergrundwissens konnte ich deshalb vermuten, dass die Ziele des Fachsprachenunterrichts neu bzw. überhaupt formuliert werden müssen und der Begriff Fachsprachenunterricht (neu) definiert werden muss. Die Frage, ob der Fachsprachenunterricht in Batumi als Gebiet mit eigenen Problemen und Anforderungen überhaupt erkannt wird, wird bei der vorlegenden Untersuchung ebenfalls nachgegangen.

Im günstigsten Fall ist die ideale Problemlösungsmöglichkeit die Zusammenarbeit mit Vertretern des entsprechenden Faches, die den geringeren fachspezifischen Wissensstand der Fremdsprachenlehrenden ausgleichen können. Dies ist besonders dann wichtig und von Nutzen, wenn aufgrund unterschiedlicher Inhaltsund Ausdrucksysteme Unklarheiten bzw. Interpretationsschwierigkeiten auftreten.Ein berufsbezogener Sprachunterricht soll unbedingt an den speziellen Bedürfnissen der jeweiligen Lernenden orientieren. Für viele Fächer sind kaum passende Lehrwerke vorhanden. In diesem Fall müssen fachspezifische Themen/Texte nach dem Grad der Spezialisierung von den Lehrenden geleistet werden. Um die passenden Lehrmaterial auszuwählen bzw. im Voraus zu bearbeiten, muss das Sprachenzentrum eng mit den Fakultäten zusammenarbeiten und ihren Anforderungen anpassen. Berufsbezogener Fremdsprachenunterricht soll auf die sprachliche Handlungsfähigkeit im Beruf vorbereiten. Aber welche fremdsprachlichen Kenntnisse und Fertigkeiten werden in dem Beruf von dem Lernenden konkret erwartet, welche sprachlichen Handlungen muss er verrichten? Um das zu erfahren, müssen sich die Lernenden und auch die Lehrenden in die Praxis begeben: Experten befragen, Betriebe besuchen, Recherchen anstellen.

Fortbildungsseminare den Fremdsprachenlehrkräfte anbieten, die in einer Ausbildung nur wenig oder gar nicht mit diesem Bereich in Berührung gekommen sind, die aber durch Veränderungen in der Ausbildungspolitik plötzlich vor der Aufgabe stehen, Berufs- und fachsprachenorientiert zu unterrichten.Eine weitere Möglichkeit für den Fremdsprachenlehrer, die Berufswirklichkeit und die fachlichen Inhalte besser kennen zu lernen, wird dadurch erreicht, das der Fremdsprachenlehrer mehr mit seinen Fachkollegen kooperiert. Um das zu fördern, können z.B. „Fortbildungsveranstaltungen“ für Kollegenpaare (Tandems) angeboten werden.

\section{Literaturverzeichnis}

Baumann, Klaus-Dieter (2000): Die Entwicklung eines integrativen Fachsprachenunterrichts - eine aktuelle Herausforderung der Angewandten Linguistik. In:Baumann, Klaus-Dieter/Kalverkämper, Hartwig/Steinberg-Rahal, Kerstin (Hg.): Sprachen im Beruf. Stand-Probleme-Perspektiven. Tübingen: Gunter Narr Verlag. S.149-173. (Forum für Fachsprachen-Forschung; Bd.38)

Buhlmann, Rosemarie/ Fearns, Anneliese (2000):Handbuch des Fachsprachenunterrichts. Tübingen: Gunter Narr Verlag.

Hoffmann, Lothar (1976): Kommunikationsmittel Fachsprache. Eine Einführung. Berlin:Akademie- Verlag. 
Bausch/ Christ/ Königs/ Krumm. (Hg.): Fremdsprachendidaktik und Sprachlehrforschung als Ausbildungs- und Forschungsdisziplinen. Ausbildung und Ausbildungsforschung als Aufgaben von Sprachlehrforschung und Fremdsprachendidaktik. Narr, Tübingen 1997. 109 - 117.

Möhn, Deiter/Pelka, Roland (1984): Fachsprachen: Eine Einführung in:Germanistische Arbeitshefte. Bd. 30. Tübingen: Niemeyer -Verlag.

Mayer, Hans Joachim (2000): hochschulpolitische Kontexte für Sprachen im Beruf. In:Baumann,klausDieter/Kalvenkemper, Hartwig/Steinberg-Rahal, Kerstin: Sprachen im Beruf. Stand-ProblemePerspektiven.Tübingen:Günter Narr Verlag. S.31-41.

Willis J. Edmondson, Juliane House (2011). Einführung in die Sprachlehrforschung. Tübingen: Narr Francke Attempto Verlag. 